\title{
The impact of mental and somatic stressors on physical activity and sedentary behaviour in adults with type 2 diabetes mellitus: a diary study
}

\author{
Louise Poppe $^{\text {Corresp., } 1}$, Annick De Paepe ${ }^{2}$, Dimitri M.L. Van Ryckeghem ${ }^{2,3,4}$, Delfien Van Dyck ${ }^{5}$, Iris Maes $^{5}$, Geert \\ Crombez $^{2}$ \\ 1 \\ ${ }^{1}$ Department of Public Health and Primary Care, Ghent University, Ghent, Belgium \\ 2 Department of Experimental Clinical and Health Psychology, Ghent University, Ghent, Belgium \\ ${ }^{3}$ Section Experimental Health Psychology, Clinical Psychological Science, Maastricht University, Maastricht, The Netherlands \\ 4 \\ 5 Department of Movement and Sports Sciences, Ghent University, Ghent, Belgium \\ Corresponding Author: Louise Poppe \\ Email address: louise.poppe@ugent.be
}

Background. Adopting an active lifestyle is key in the management of type 2 diabetes mellitus (T2DM). Nevertheless, the majority of individuals with T2DM fails to do so. Additionally, individuals with T2DM are likely to experience mental (e.g. stress) and somatic (e.g. pain) stressors. Research investigating the link between these stressors and activity levels within this group is largely lacking. Therefore, current research aimed to investigate how daily fluctuations in mental and somatic stressors predict daily levels of physical activity (PA) and sedentary behaviour among adults with T2DM.

Methods. Individuals with T2DM ( $\mathrm{N}=54)$ were instructed to complete a morning diary assessing mental and somatic stressors and to wear an accelerometer for 10 consecutive days. The associations between the mental and somatic stressors and participants' levels of PA and sedentary behaviour were examined using (generalized) linear mixed effect models.

Results. Valid data were provided by 38 participants. We found no evidence that intra-individual increases in mental and somatic stressors detrimentally affected participants' activity levels. Similarly, levels of sedentary behaviour nor levels of PA were predicted by inter-individual differences in the mental and somatic stressors. 


\section{The impact of mental and somatic stressors on physical}

2 activity and sedentary behaviour in adults with type 2

3 diabetes mellitus: a diary study

6 Louise Poppe ${ }^{1}$, Annick De Paepe ${ }^{2}$, Dimitri Van Ryckeghem ${ }^{2,3,4}$, Delfien Van Dyck ${ }^{5}$, Iris Maes ${ }^{5}$,

7 Geert Crombez ${ }^{2}$

8

$9 \quad{ }^{1}$ Department of Public Health and Primary Care, Ghent University, Ghent, Belgium

$10{ }^{2}$ Department of Experimental Clinical and Health Psychology, Ghent University, Ghent,

11 Belgium

$12{ }^{3}$ Section Experimental Health Psychology, Clinical Psychological Science, Maastricht

13 University, Maastricht, The Netherlands

$14{ }^{4}$ Department of Behavioural and Cognitive Sciences, University of Luxembourg, Luxembourg

15 City, Luxembourg

$16{ }^{5}$ Department of Movement and Sports Sciences, Ghent University, Ghent, Belgium

19 Corresponding Author:

20 Louise Poppe ${ }^{1}$

21 Corneel Heymanslaan 10, 9000 Ghent, East-Flanders, Belgium

22 Email address: louise.poppe@ugent.be 


\section{Abstract}

25 Background. Adopting an active lifestyle is key in the management of type 2 diabetes mellitus

26 (T2DM). Nevertheless, the majority of individuals with T2DM fails to do so. Additionally,

27 individuals with T2DM are likely to experience mental (e.g. stress) and somatic (e.g. pain)

28 stressors. Research investigating the link between these stressors and activity levels within this

29 group is largely lacking. Therefore, current research aimed to investigate how daily fluctuations

30 in mental and somatic stressors predict daily levels of physical activity (PA) and sedentary

31 behaviour among adults with T2DM.

32 Methods. Individuals with T2DM $(\mathrm{N}=54)$ were instructed to complete a morning diary assessing

33 mental and somatic stressors and to wear an accelerometer for 10 consecutive days. The

34 associations between the mental and somatic stressors and participants' levels of PA and

35 sedentary behaviour were examined using (generalized) linear mixed effect models.

36 Results. Valid data were provided by 38 participants. We found no evidence that intra-individual

37 increases in mental and somatic stressors detrimentally affected participants' activity levels.

38 Similarly, levels of sedentary behaviour nor levels of PA were predicted by inter-individual

39 differences in the mental and somatic stressors. 


\section{Introduction}

42 Diabetes mellitus is a significant problem in western society and the number of individuals with

43 diabetes mellitus is still growing (IDF 2019). In 2019, 8.9\% of European adults lived with

44 (un)diagnosed diabetes mellitus (IDF 2019). The most common form of diabetes mellitus is type

452 diabetes mellitus (T2DM), accounting for $90 \%$ of all diabetes cases (IDF 2019). Adopting and

46 maintaining an active lifestyle, with sufficient physical activity (PA) and a limited amount of

47 sitting time, is considered key in the prevention and management of T2DM (Dempsey et al.

48 2016; Sigal et al. 2006). Similar to the general population, individuals with T2DM are

49 recommended to accumulate 150-300 minutes of moderate-intensity PA; or 75-150 minutes of

50 vigorous-intensity PA; or an equivalent combination of moderate- and vigorous-intensity PA

51 throughout the week and to do muscle-strengthening activities on two or more days per week

52 (WHO 2020). Furthermore, adults with T2DM are encouraged to minimize the amount of time spent being sedentary (WHO 2020) and to interrupt prolonged sitting time with bouts of lightintensity PA (LPA) every 30 minutes (Colberg et al. 2016). Yet, despite this knowledge, the majority of patients fails to reach the predefined health guidelines regarding PA and accumulates high levels of sitting time (Hamer et al. 2013; Morrato et al. 2007).

In contrast with other preventive health behaviours (e.g., getting a flu shot), adopting and maintaining an active lifestyle requires daily effort and continued dedication. These efforts are therefore likely to be influenced by daily variations in how we feel and interact with our environment (Dunton 2017). Indeed, individuals act in the context of highly variable levels of mental (e.g., negative affect) and somatic (e.g., pain or fatigue) stressors (Kanning \& Schoebi 2016). Understanding the impact of these stressors upon activity behaviours is particular of interest for developing interventions that provide in time support to act upon the possibly detrimental impact of mental and somatic stressors (e.g. Just-In-Time-Adaptive-Interventions (Nahum-Shani et al. 2017)). For example, the mobile application "OnTrack" identifies potential triggers for dietary lapses (including negative mood or fatigue) and provides users with information about potential ways to cope with the detected triggers (Forman et al. 2019; Goldstein et al. 2017). Currently, this type of innovative interventions does not exist within the domain of active lifestyle adoption and maintenance (Hardeman et al. 2019). A key reason for a lack of similar interventions in this domain at least partially relates to a lack of understanding how mental and somatic stressors impact upon people's daily activity levels. To support future

73

74

75

76

77

78

79

80 development of interventions that are tailored to the momentary state (mental and somatic) of an individual, it is key to understand how mental and somatic stressors impact upon people's activity levels, especially within populations who are often experiencing these stressors. Individuals with T2DM often experience mental and somatic stressors in daily life (Fritschi \& Quinn 2010; Heidari et al. 2019). In comparison with the general population, people with T2DM are more likely to experience a major depressive episode (Darwish et al. 2018) and to experience fatigue and pain (Heidari et al. 2019). As yet, no research has examined whether within-person alterations in mental and somatic stressors affect activity levels throughout the day in individuals with T2DM. 
81 A few studies in non-patient populations have already examined how intra-individual changes in

82

83

84

85

86

87

88

89

90

91

92

93

94

95

96

97

98

99

100

101

102

103

104

105

106

107

108

109

110

111

112

113

114

115

116

117

118

119

mental and somatic stressors influence people's activity levels. Dunton and colleagues demonstrated that, within adults aged 50 years and above, momentary negative affect predicted lower levels of self-reported moderate-to-vigorous PA (MVPA) in the subsequent 4 hours interval after the assessment (Dunton et al. 2010). Similarly, Zenk and colleagues found that negative affect measured in the morning was associated with lower levels of subsequent daily accelerometer-measured MVPA and higher levels of subsequent daily accelerometer-measured sedentary behaviour in African-American women aged 25-64 years (Zenk et al. 2017). Elevated levels of momentary stress and fatigue have also been found to decrease subsequent levels of accelerometer-measured PA in adults (Jones et al. 2017; Liao et al. 2017; Vetrovsky et al. 2021). Yet, studies examining the impact of intra-individual fluctuations in mental and somatic stressors on activity levels within patient populations, however, are scarce. Research in people with fibromyalgia and/or chronic fatigue syndrome showed that momentary pain and fatigue predicted decreased accelerometer-measured activity levels in the 30 minute interval after the assessment (Kop et al. 2005). Similarly, Murphy and colleagues found that increased momentary fatigue predicted reduced levels of accelerometer-assessed PA in the subsequent 4 hours among people with symptomatic knee and hip osteoarthritis (Murphy et al. 2012).

The aim of the current study is to address the research gap regarding the daily impact of mental and somatic stressors on the activity levels of people with T2DM. More specifically, we aimed to assess the influence of intra-individual variations in mental and somatic stressors on daily levels of accelerometer-measured PA and sedentary behaviour in individuals with T2DM.

\section{Materials \& Methods}

\section{Participants}

Data-collection for this study was part of the baseline test of a randomized controlled trial examining the efficacy of an online intervention promoting an active lifestyle (Poppe et al. 2019b). Consequently, the a priori power analysis was based on the targeted outcomes of the randomized controlled trial (Poppe et al. 2019a). Based on this power analysis we aimed to recruit 96 people with T2DM. Individuals with T2DM were recruited via different channels. First, people with T2DM were recruited via the Ghent University Hospital and the Damian General Hospital (Ostend). Because recruitment via the hospitals was slower than expected, the study was also advertised via the Flemish Diabetes Association. Finally, people with T2DM who participated in previous research (Poppe et al. 2017) were invited to participate in the randomized controlled trial. To be eligible, patients had to (1) be diagnosed with T2DM for at least one month, (2) be 18 years or older, (3) be Dutch-speaking, (4) be computer-literate and (5) have Internet access. The study was conducted between January and August 2018. The study was approved by the Committee of Medical Ethics of the Ghent University Hospital (Belgian registration number: B670201732566). The protocol for the trial was published (Poppe et al. 2019a) and all participants provided a written informed consent. 
120

121

122

123

124

125

126

127

128

129

130

131

132

133

134

135

136

137

138

139

140

141

142

143

144

145

146

147

148

149

150

151

152

153

154

155

156

157

158

159

\section{Procedure}

After enrolment, participants were visited at home by a researcher. Participants' weight and waist circumference were assessed. Next, participants were instructed to complete an ad-hoc questionnaire assessing demographic information. Finally, they were instructed to wear an accelerometer and to fill out an online morning diary for 10 consecutive days starting the day after the home visit. After this period of 10 days, the accelerometer was recollected and participants were randomized to the intervention group or the waiting-list control group.

\section{Measurements}

\section{Demographic information}

Participants' age, sex, height, civil status, level of education, profession and time since diagnosis were assessed via an ad hoc questionnaire. Level of education was dichotomized in "low" (primary or secondary education) versus "high" (college or university). A Seca weighting scale (type 813) and a Seca measuring tape were used to determine participants' weight and waist circumference. To minimize measurement error, participants' weight and waist circumference were assessed twice. In case there was a difference larger than 100 grams or $1 \mathrm{~cm}$, the measurement was conducted a third time. The mean of the measurements was considered as the final score.

\section{Mental wellbeing}

During the home visit, participants' levels of anxiety and depression were assessed using scales of the Patient-Reported Outcomes Measurement Information System (PROMIS) (Cella et al. 2010). The depression short-form scale (version 1.0) and the anxiety short-form scale (version 1.0) each contain six questions with five answer options (i.e. "never", "seldom", "sometimes", "often" and "always") and assess feelings of anxiety and depression in the past seven days.

\section{Activity levels}

Participants' levels of PA and sedentary behaviour were assessed using ActiGraph accelerometers (type GT3X+) worn on the right hip. The validity and reliability of accelerometers are influenced by the selected cut points for data reduction. As our sample's mean age was 63 years (see Results), cut points for older adults described by Barnett et al. (Barnett et al. 2016) were used to categorize each minute of wear time as sedentary (0-25 counts per minute (CPM)), LPA (26-1012 CPM) or MVPA ( $\geq 1013 \mathrm{CPM})$. In the validation study of Barnett et al. older adults were instructed to wear the GT3X+ accelerometer and a GPS monitor on the right hip during overground walking. Participants' energy expenditure was assessed using a mobile breath-by-breath gas analysis system while they walked at different walking speeds. Similarly, Aguilar-Farías et al. (2013) found that the hip-worn ActiGraph GT3X showed good accuracy for detecting sedentary behavior in older adults in free-living environments using the $<25$ CPM cut point. In this study the ActiGraph GT3X was compared with an inclinometer (i.e. the ActivPal) (Aguilar-Farías et al. 2014). Finally, Aadland and Ylvisåker (2015) showed that the 
160 hip-worn ActiGraph GT3X is a reliable tool for assessing intensity-specific PA and sedentary

161

162

163

164

165

166

167

168

169

170

171

172

173

174

175

176

177

178

179

180

181

182

183

184

185

186

187

188

189

190

191

192

193

194

195 days (Aadland \& Ylvisåker 2015).

Participants were instructed to wear the accelerometer during waking hours and to remove the device for water-based activities (e.g., bathing). The accelerometer was initialised and the data were processed using ActiLife 6.13.3 software (ActiGraph, Fort Walton Beach, FL, USA). An epoch was set at 60 seconds and periods of more than 60 minutes of consecutive 0 counts were considered as non-wear time (Troiano et al. 2008). A valid day was considered as a day with a minimum of 600 minutes of wear time (Troiano et al. 2008). For each participant the number of minutes per day spent sedentary, performing LPA and performing MVPA were calculated.

\section{Daily mental and somatic stressors}

The morning diary was created with the survey software LimeSurvey (LimeSurvey Project, Hamburg, Germany). All participants received an e-mail with a link to the website and a unique token to $\log$ in. Participants filled out the morning diary using their personal computer or tablet and could only access and complete the morning diary between 3 AM and 11 AM. The morning diary assessed mental and somatic stressors that are prevalent in individuals with T2DM (i.e. fatigue (Fritschi \& Quinn 2010; Heidari et al. 2019), stress (Hackett \& Steptoe 2017; Qiu et al. 2017), sadness (Ali et al. 2006; Darwish et al. 2018), pain (Heidari et al. 2019; Kirk et al. 2019), nausea/dizziness (Heidari et al. 2019) and numbness/tingling in the limbs (Aikens 1998; Kästenbauer et al. 2004)). All mental and somatic stressors were assessed using single-item measures. This is in line with numerous diary studies (e.g. Bouwmans et al. (2017)) and was done to limit the time needed to complete the diary and hence minimise the chances of drop-out or random responses. Additionally, considering the repeated assessment in daily life, traditional questionnaires assessing mental and somatic stressors are not by default valid for this type of research (Degroote et al. 2020). Participants were instructed to indicate how strongly they experienced the stressor "right now" using a 10-point scale, ranging from 1 "(absolutely not)" to 10 "(very much)".

To ensure comprehensibility of the items assessing the mental and somatic stressors, a cognitive interview was conducted with four volunteers (mean age $=58.3$ years $(\mathrm{SD}=6.5) ; 75 \%$ women; $50 \%$ with a high level of education (i.e. college/university); $50 \%$ diagnosed with T2DM) (Beatty $\&$ Willis 2007; Poppe et al. 2019a). For these cognitive interviews, the volunteers were instructed to answer each item of the morning diary and to explain how they came to this answer. Several adaptations were performed to the initial items, assuring the unambiguous comprehension of the items assessing the mental and somatic stressors. To avoid possible interference, these volunteers did not take part in the current study. 
196

197

198

199

200

201

202

203

204

205

206

207

208

209

210

211

212

213

214

215

216

217

218

219

220

221

222

223

224

225

226

227

228

229

230

231

232

233

234

\section{Data-analysis}

The data were analysed using R version 3.2.5 (R Development Core Team 2010). To ensure the quality of the data, participants' data were only included when they (1) filled-out the diary for a minimum of 7 days (Rost et al. 2016; Van Ryckeghem et al. 2013) and (2) had a minimum of 4 valid accelerometer days (Van Dyck et al. 2019).

To take into account the clustering of the data within participants (generalized) linear mixed effect models as implemented in the package lme4 version 1.1-19 (Bates et al. 2014) were used to analyse the data. Considering inter- and intra-individual differences in accelerometer wear time, participants' number of daily minutes spent performing sedentary behaviour, LPA or MVPA were divided by the daily number of minutes that participants had worn the accelerometer (Hooker et al. 2016). Levels of within- and between-subject variance of the stressors and the activity levels were calculated by running intercept-only models (i.e. models only including a fixed and random intercept) with each of the stressors as well as participants' levels of sedentary behaviour, LPA and MVPA as outcome variable.

To examine the effect of the stressors on participants' levels of sedentary behaviour, LPA and MVPA, (generalized) linear mixed effect models were fitted with the mental and somatic stressors as between-subject (i.e. mean of the variable at the subject-level) as well as withinsubject (i.e. individuals' daily score minus their mean score) variables. This was done because the standard mixed model approach does not distinguish between these within- and betweencluster effects and implicitly assumes these effects are the same. However, incorrectly assuming common effects can obscure the association of covariates with the response. By modelling the within-and between-subject effects separately, the discrepancy of these effects becomes explicit (see also Neuhaus \& Kalbfleisch (1998)). Participants' age, sex (i.e. male vs. female), waist circumference, level of education (i.e. low vs. high), retirement status (i.e. retired vs. not retired), level of anxiety (PROMIS) and level of depression (PROMIS) were also included in the model. To facilitate convergence, the variables "age", "waist circumference", "level of anxiety (PROMIS)" and "level of depression (PROMIS)" were standardized before they were entered in the model.

For each fitted model the normality assumption was checked by visually inspecting the residuals versus fitted values plot and the quantile-quantile plot. If normality could not be assumed, the Bayesian Information Criterion (BIC) of models with different variance and link functions (i.e., Gaussian with identity, Gamma with log, Gamma with identity, Poisson with log, and negative binomial with log) were compared and the model with the lowest BIC value was selected (Germeys \& De Gieter 2018).

\section{Results}

Data availability and descriptive statistics

Despite the intensive recruitment process, only 54 adults with T2DM agreed to participate in the study. After correcting for the required number of valid accelerometer and diary days, data of 39 
235

236

237

238

239

240

241

242

243

244

245

246

247

248

249

250

251

252

253

254

255

256

257

258

259

260

261

262

263

264

265

266

267

268

269

270

271

272

273

participants was retained. Supplementary File 1 provides a side-by-side comparison of the characteristics of participants who provided valid accelerometer and diary data and those who did not. Demographic information of one participant was missing. Consequently, analyses were performed on the data of 38 participants. Table 1 provides participants' demographic characteristics.

[insert Table 1 about here]

On average, participants wore the accelerometer for 9 days ( $\mathrm{SD}=2)$ and completed the diary on 9 days $(\mathrm{SD}=1)$. Table 2 displays participants' mean levels of PA and sedentary behaviour as well as the mean scores on the mental and somatic stressors. Five people did not reach the current PA guidelines (i.e. $<150$ minutes of MVPA per week). Supplementary File 2 illustrates the individual scores on the mental and somatic stressors over the measurement period.

[insert Table 2 about here]

Associations between mental and somatic stressors and activity levels

Table 3 presents the results of the analysis for sedentary behaviour, LPA and MVPA. For sedentary behaviour a Gaussian model with an identity link was used. A Gamma model with an identity link function was used to assess the effect of the stressors on participants' levels of LPA and MVPA. Intra-individual nor inter-individual differences in the stressors (i.e., fatigue, stress, pain, nausea/dizziness, numbness/tingling, and sadness) were found to be associated with alterations in participants' sedentary behaviour, LPA or MVPA ( $\mathrm{p}>.05)$.

[insert Table 3 about here]

\section{Discussion}

This study investigated the impact of mental and somatic stressors, assessed in the morning, on accelerometer-measured PA and sedentary behaviour during that day in individuals with T2DM. Changes in the mental and somatic stressors across days were not found to be associated with changes in participants' levels of sedentary behaviour, LPA or MVPA. Similarly, levels of sedentary behaviour, LPA, and MVPA were not predicted by between-subject differences in the mental and somatic stressors.

This pattern of results was surprising. We had expected that both mental and somatic stressors would be an obstacle for participants' activity levels during that day. Individuals with T2DM may experience a variety of mental and somatic stressors, such as sadness (Darwish et al. 2018) and pain (Heidari et al. 2019), and some of these have been associated with more sedentary behaviour and low levels of PA. For example, Chastin et al. found that pain was one of the main drivers for being sedentary, albeit not in a sample of patients with T2DM (Chastin et al. 2014).

PeerJ reviewing PDF | (2021:01:57126:1:0:CHECK 30 Apr 2021) 
274 There are at least three explanations for the current results.

275 First, we may not have detected effects because of a limited sample size. However, simulation 276 studies have shown that a minimum of 30 clusters (in this case a minimum of 30 participants) is 277 needed to obtain unbiased point estimates of the level 1 and level 2 fixed effects (i.e. the level of 278 the stressor within (level 1) and between (level 2) subjects) and the fixed standard errors 279 (McNeish \& Stapleton 2016). Considering that 38 participants provided valid data, we consider 280 it unlikely that a lack of power explains our results.

281 Second, the mental and somatic stressors assessed in the morning may have no or a limited effect 282 on participants' activity levels. It may well be that participants had a high maintenance self283 efficacy, meaning that they were able to remain physically active in the presence of stressors 284 (Schwarzer 2008). If this is the case, it would be informative to examine how participants 285 achieve this.

286 Third, the lack of effect detected might be attributed to the limited variability in the reported 287 stressors. The majority of the participants reported not to experience the stressors

"nausea/dizziness" and "numbness/tingling" when completing the diary (see Supplementary File 2). Indeed, it is possible that variability in these stressors might only be relevant for a subgroup of people with T2DM (e.g., people with neuropathy). Similarly, limited variation over days was

292

293

294

295

296

297

298

299

300

301

302

303

304

305

306

307

308

309

310

311

312

313 found for the stressor "sadness" as most participants reported not to experience this stressor when completing the diary. Röcke et al. instructed 19 older adults to rate their positive and negative affect for a period of 45 days. Similarly, the mean score for negative affect was low (i.e. 1.32 on 8 ) and the majority of participants (i.e. 13 out of 19) provided the same rating score for "sad" on more than $90 \%$ of the 45 days (Röcke et al. 2009).

More variation over days was found for the stressors "fatigue", "stress" and "pain".

Nevertheless, no evidence for an association between these stressors and participants' activity levels was found. Vetrovsky et al. (2021) assessed older adults' morning fatigue and daily PA for a period of four weeks and found that greater morning fatigue was associated with less accelerometer-measured daily MVPA (Vetrovsky et al. 2021). However, in this study depressive symptoms, which might influence the stressor (i.e. fatigue) as well as the outcome (i.e. levels of sedentary behaviour and PA) were not taken into account. Furthermore, in contrast with our study, the within- and between-subject effects were not separately modelled. Similar to our results, Liao et al. found no between- or within-person association between levels of fatigue and levels of LPA and MVPA in the subsequent $30 \mathrm{~min}$. time window among healthy adults (mean age $=40.4$ ) (Liao et al. 2017). However, in the study of Liao et al. daily increases in negative affect (including feelings of depression and stress) did predict an increase in LPA in the subsequent $30 \mathrm{~min}$. time window. Nevertheless, it should be noted that mean levels of negative affect were higher in their study (i.e., 1.44 on a 5-point scale) than in ours (i.e., 1.37 (sadness) and 1.81 (stress) on a 10-point scale). In the current study the reported levels of the mental and somatic stressors were overall low (i.e. the mean score ranged between 1.21 (nausea/dizziness) and 2.34 (fatigue) on 10). Consequently, the level of these stressors might have been too low to actually influence participants' levels of sedentary behaviour, LPA or MVPA. A first potential 
314 explanation for these low scores within our sample is self-selection: individuals with lower levels 315 of mental or somatic stressors might be more likely to participate in an activity-promoting 316 programme than individuals facing higher levels of these stressors. The low scores for the 317 stressors "fatigue", "stress" and "pain" can also be explained by the timing of the diary (i.e. in 318 the morning). It is possible that the intensity of these stressors is relatively low when waking up, 319 but increases throughout the day. Similarly, Vetrovsky et al. reported low levels of morning

320

321

322

323

324

325

326

327

328

329

330

331

332

333

334

335

336

337

338

339

340

341

342

343

344

345

346

347

348

349

350

351

352

353 fatigue in their study focusing on the relation between morning fatigue and PA among older adults (Vetrovsky et al. 2021). Further research implementing multiple measurements throughout the day is needed to elucidate these findings.

This study has several strengths. First, according to our knowledge, this is the first study examining the within-subject effects of mental and somatic stressors on physical activity and sedentary behaviour in individuals with T2DM. Second, participants' activity levels were assessed using accelerometers rather than self-report measures. In doing so the impact of recall biases was minimized. Third, completion rates of the morning diary and adherence to the accelerometer protocol were high. On average, participants filled out the diary and wore an accelerometer on 9 of the 10 days.

There are also a number of limitations. First, according to the accelerometer data, participants' activity levels were already quite high (i.e. $49 \mathrm{~min}$./day of MVPA on average). However, the cut points for classifying participants' activity levels are based on research with older adults having a mean age of 70 years (range: $60-87.6$ years) (Barnett et al. 2016). Because the mean age of our sample was 63 years (range: $50-81$ years), it is possible that we overestimated participants' activity levels. When applying the Freedson cut points for adults (Freedson et al. 1998), our sample accumulates on average 21 minutes of MVPA per day and 19 participants do not meet the current PA guidelines (data not shown). Furthermore, it is important to note that our main interest was to investigate the relation between mental and somatic stressors and activity levels rather than participants' mean activity levels. Second, the mental and somatic stressors were only measured in the morning. These data were then used to predict participants' activity levels over that day. Other studies investigating shorter time frames (e.g. the impact of stressors on activity levels in the following 15 minutes (Jones et al. 2017), 30 minutes (Kop et al. 2005) or 4 hours (Dunton et al. 2010; Murphy et al. 2012) after the measurement of the stressor) found more evidence for within-subject associations between mental and/or somatic stressors and people's activity levels. Indeed, our lengthy time frame (i.e. one day) might have masked short-term effects of intra-individual increases in the mental and somatic stressors. More advanced designs including ecological momentary assessment with multiple measurements throughout the day may overcome this problem. However, as this study was part of the baseline test of a randomized controlled trial, it was decided not to overwhelm participants with a more intensive design of data collection. Third, although we aimed to adjust the analysis for the most relevant confounding variables, confounding from other variables remains a possibility. For example, medication adherence was not assessed in the current study. Hence, we were unable to adjust the analysis for medication effects. Finally, the participants of this study had agreed to take part in a 
354 randomized controlled trial testing the effect of an online intervention targeting an active

355 lifestyle and were only included when they met the inclusion criteria. It is therefore possible that

356 the participants differed on several domains from people who refused to participate (e.g., age or

357 severity of diabetes-related complications). As self-selection might have occurred, one should be

358 cautious to extrapolate our results to the overall population of adults with T2DM.

359 The current findings have implications for intervention development as well as for further

360 research. In order to be relevant targets for interventions tailored to the momentary state of

361 individuals, mental or somatic stressors should (1) vary over time and (2) predict individuals'

362 activity levels. In line with previous research (Röcke et al. 2009), we found limited variation

363 over days for the stressor "sadness". Hence, this stressor might be of limited interest for the

364 development of interventions tailored to the momentary state of people with T2DM. Higher

365 variation over days was detected for the stressors "fatigue", "stress" and "pain". However,

366 considering the lack of evidence found for an association between these stressors and

367 participants' activity levels, interventions targeting these stressors might have limited impact.

368 Two somatic stressors, namely "numbness/tingling" and "nausea/dizziness" only varied within a

369 subgroup of our sample. Further research is needed to examine the impact of these stressors in

370 specific subgroups of people with T2DM (e.g., people with neuropathy).

371

\section{Conclusions}

373 The aim of this study was to investigate whether fluctuations in mental and somatic stressors

374 across days affect daily levels of PA and sedentary behaviour in adults with T2DM. No

375 detrimental effect of intra-individual increases in mental and somatic stressors on participants'

376 activity levels was detected. Similarly, levels of sedentary behaviour, LPA, nor MVPA were

377 predicted by between-subject differences in the mental and somatic stressors. This study is the

378 first to investigate within-subject effects of mental and somatic stressors on physical activity and

379 sedentary behaviour in individuals with T2DM. Studies adopting ecological momentary

380 assessment with multiple measurements throughout the day are needed to gain more insight into

381 the potential short-term effects of mental and somatic stressors on the activity levels of people

382 with T2DM. 
383

384

385

386

387

388

389

390

391

392

393

394

395

396

397

398

399

400

401

402

403

404

405

406

407

408

409

410

411

412

413

414

415

416

417

418

419

420

421

422

423

424

425

426

427

\section{References}

Aadland E, and Ylvisåker E. 2015. Reliability of the Actigraph GT3X+ accelerometer in adults under free-living conditions. Plos One 10:e134606.

Aguilar-Farías N, Brown WJ, and Peeters GMEE. 2014. ActiGraph GT3X+ cut-points for identifying sedentary behaviour in older adults in free-living environments. Journal of Science and Medicine in Sport 17:293-299. http://dx.doi.org/10.1016/j.jsams.2013.07.002

Aikens JE. 1998. Prevalence of somatic indicators of distress in diabetes patients: Comparison to psychiatric patients and community nonpatients. The International Journal of Psychiatry in Medicine 28:265-272.

Ali S, Stone MA, Peters JL, Davies MJ, and Khunti K. 2006. The prevalence of co-morbid depression in adults with Type 2 diabetes: a systematic review and meta-analysis. Diabetic Medicine 23:1165-1173. 10.1111/j.1464-5491.2006.01943.x

Barnett A, van den Hoek D, Barnett D, and Cerin E. 2016. Measuring moderate-intensity walking in older adults using the ActiGraph accelerometer. BMC geriatrics 16:211.

Bates D, Mächler M, Bolker B, and Walker S. 2014. Fitting linear mixed-effects models using lme4. arXiv preprint arXiv: 14065823.

Beatty PC, and Willis GB. 2007. Research synthesis: The practice of cognitive interviewing. Public Opinion Quarterly 71:287-311.

Bouwmans ME, Bos EH, Hoenders HR, Oldehinkel AJ, and de Jonge P. 2017. Sleep quality predicts positive and negative affect but not vice versa. An electronic diary study in depressed and healthy individuals. Journal of Affective Disorders 207:260-267.

Cella D, Riley W, Stone A, Rothrock N, Reeve B, Yount S, Amtmann D, Bode R, Buysse D, Choi S, Cook K, DeVellis R, DeWalt D, Fries JF, Gershon R, Hahn EA, Lai J-S, Pilkonis P, Revicki D, Rose M, Weinfurt K, and Hays R. 2010. The Patient-Reported Outcomes Measurement Information System (PROMIS) developed and tested its first wave of adult self-reported health outcome item banks: 2005-2008. Journal of Clinical Epidemiology 63:1179-1194. http://dx.doi.org/10.1016/j.jclinepi.2010.04.011

Chastin SF, Fitzpatrick N, Andrews M, and DiCroce N. 2014. Determinants of sedentary behavior, motivation, barriers and strategies to reduce sitting time in older women: a qualitative investigation. International journal of environmental research and public health 11:773-791.

Colberg SR, Sigal RJ, Yardley JE, Riddell MC, Dunstan DW, Dempsey PC, Horton ES, Castorino K, and Tate DF. 2016. Physical Activity/Exercise and Diabetes: A Position Statement of the American Diabetes Association. Diabetes Care 39:2065-2079. $10.2337 / \mathrm{dc} 16-1728$

Darwish L, Beroncal E, Sison MV, and Swardfager W. 2018. Depression in people with type 2 diabetes: current perspectives. Diabetes, metabolic syndrome and obesity : targets and therapy 11:333-343. 10.2147/DMSO.S106797

Degroote L, DeSmet A, De Bourdeaudhuij I, Van Dyck D, and Crombez G. 2020. Content validity and methodological considerations in ecological momentary assessment studies on physical activity and sedentary behaviour: a systematic review. International Journal of Behavioral Nutrition and Physical Activity 17:1-13.

Dempsey PC, Owen N, Yates TE, Kingwell BA, and Dunstan DW. 2016. Sitting Less and Moving More: Improved Glycaemic Control for Type 2 Diabetes Prevention and Management. Curr Diab Rep 16:114. 10.1007/s11892-016-0797-4 
428 Dunton GF. 2017. Ecological Momentary Assessment in Physical Activity Research. Exercise

429

430

431

432

433

434

435

436

437

438

439

440

441

442

443

444

445

446

447

448

449

450

451

452

453

454

455

456

457

458

459

460

461

462

463

464

465

466

467

468

469

470

471

472

473

and Sport Sciences Reviews 45:48-54. 10.1249/JES.0000000000000092

Dunton GF, Atienza AA, Castro CM, and King AC. 2010. Using Ecological Momentary Assessment to Examine Antecedents and Correlates of Physical Activity Bouts in Adults Age 50+ Years: A Pilot Study. Annals of Behavioral Medicine 38:249-255. 10.1007/s12160-009-9141-4

Forman EM, Goldstein SP, Zhang F, Evans BC, Manasse SM, Butryn ML, Juarascio AS, Abichandani P, Martin GJ, and Foster GD. 2019. OnTrack: Development and feasibility of a smartphone app designed to predict and prevent dietary lapses. Translational behavioral medicine 9:236-245.

Freedson PS, Melanson E, and Sirard J. 1998. Calibration of the Computer Science and Applications, Inc. accelerometer. Medicine and Science in Sports and Exercise 30:777781.

Fritschi C, and Quinn L. 2010. Fatigue in patients with diabetes: A review. Journal of Psychosomatic Research 69:33-41. https://doi.org/10.1016/j.jpsychores.2010.01.021

Germeys L, and De Gieter S. 2018. A diary study on the role of psychological detachment in the spillover of self-control demands to employees' ego depletion and the crossover to their partner. European Journal of Work and Organizational Psychology 27:140-152. 10.1080/1359432X.2017.1417259

Goldstein SP, Evans BC, Flack D, Juarascio A, Manasse S, Zhang F, and Forman EM. 2017. Return of the JITAI: Applying a Just-in-Time Adaptive Intervention Framework to the Development of m-Health Solutions for Addictive Behaviors. International journal of behavioral medicine 24:673-682. 10.1007/s12529-016-9627-y

Hackett RA, and Steptoe A. 2017. Type 2 diabetes mellitus and psychological stress - a modifiable risk factor. Nature Reviews Endocrinology 13:547-560. 10.1038/nrendo.2017.64

Hamer M, Bostock S, Hackett R, and Steptoe A. 2013. Objectively assessed sedentary time and type 2 diabetes mellitus: a case-control study. Diabetologia 56:2761-2762. 10.1007/s00125-013-3051-5

Hardeman W, Houghton J, Lane K, Jones A, and Naughton F. 2019. A systematic review of justin-time adaptive interventions (JITAIs) to promote physical activity. International Journal of Behavioral Nutrition and Physical Activity 16:31.

Heidari Z, Feizi A, Keshteli AH, Afshar H, Roohafza H, and Adibi P. 2019. Psychosomatic complaints profile in patients with type 2 diabetes: a matched case-control study. The Egyptian Journal of Neurology, Psychiatry and Neurosurgery 55:53.

Hooker SP, Hutto B, Zhu W, Blair SN, Colabianchi N, Vena JE, Rhodes D, and Howard VJ. 2016. Accelerometer measured sedentary behavior and physical activity in white and black adults: the REGARDS study. Journal of Science and Medicine in Sport 19:336341.

IDF. 2019. International Diabetes Federation Diabetes Atlas. Brussels.

Jones M, Taylor A, Liao Y, Intille SS, and Dunton GF. 2017. Real-time subjective assessment of psychological stress: Associations with objectively-measured physical activity levels. Psychology of sport and exercise 31:79-87.

Kanning MK, and Schoebi D. 2016. Momentary Affective States Are Associated with Momentary Volume, Prospective Trends, and Fluctuation of Daily Physical Activity. Frontiers in Psychology 7. 10.3389/fpsyg.2016.00744

PeerJ reviewing PDF | (2021:01:57126:1:0:CHECK 30 Apr 2021) 
474 Kästenbauer T, Irsigler P, Sauseng S, Grimm A, and Prager R. 2004. The prevalence of

475

476

477

478

479

480

481

482

483

484

485

486

487

488

489

490

491

492

493

494

495

496

497

498

499

500

501

502

503

504

505

506

507

508

509

510

511

512

513

514

515

516

517

518

519 symptoms of sensorimotor and autonomic neuropathy in Type 1 and Type 2 diabetic subjects. Journal of Diabetes and its Complications 18:27-31. https://doi.org/10.1016/S1056-8727(03)00071-0

Kirk JK, Hunter JC, Mihalko SL, Danhauer SC, and Shumaker SA. 2019. Perspectives of pain in patients with type 2 diabetes. Expert Review of Endocrinology \& Metabolism 14:215219. 10.1080/17446651.2019.1592674

Kop WJ, Lyden A, Berlin AA, Ambrose K, Olsen C, Gracely RH, Williams DA, and Clauw DJ. 2005. Ambulatory monitoring of physical activity and symptoms in fibromyalgia and chronic fatigue syndrome. Arthritis \& Rheumatism 52:296-303. 10.1002/art.20779

Liao Y, Chou C-P, Huh J, Leventhal A, and Dunton G. 2017. Examining acute bi-directional relationships between affect, physical feeling states, and physical activity in free-living situations using electronic ecological momentary assessment. Journal of behavioral medicine 40:445-457. 10.1007/s10865-016-9808-9

McNeish DM, and Stapleton LM. 2016. The effect of small sample size on two-level model estimates: A review and illustration. Educational Psychology Review 28:295-314.

Morrato EH, Hill JO, Wyatt HR, Ghushchyan V, and Sullivan PW. 2007. Physical activity in U.S. adults with diabetes and at risk for developing diabetes, 2003. Diabetes Care 30:203-209. 10.2337/dc06-1128

Murphy S, Kratz A, Williams D, and Geisser M. 2012. The Association between Symptoms, Pain Coping Strategies, and Physical Activity Among People with Symptomatic Knee and Hip Osteoarthritis. Frontiers in Psychology 3. 10.3389/fpsyg.2012.00326

Nahum-Shani I, Smith SN, Spring BJ, Collins LM, Witkiewitz K, Tewari A, and Murphy SA. 2017. Just-in-time adaptive interventions (JITAIs) in mobile health: key components and design principles for ongoing health behavior support. Annals of Behavioral Medicine 52:446-462.

Neuhaus JM, and Kalbfleisch JD. 1998. Between-and within-cluster covariate effects in the analysis of clustered data. Biometrics:638-645.

Poppe L, De Bourdeaudhuij I, Verloigne M, Degroote L, Shadid S, and Crombez G. 2019a. A Self-Regulation-Based eHealth and mHealth Intervention for an Active Lifestyle in Adults With Type 2 Diabetes: Protocol for a Randomized Controlled Trial. JMIR research protocols 8:e12413.

Poppe L, De Bourdeaudhuij I, Verloigne M, Shadid S, Van Cauwenberg J, Compernolle S, and Crombez G. 2019b. Efficacy of a Self-Regulation-Based Electronic and Mobile Health Intervention Targeting an Active Lifestyle in Adults Having Type 2 Diabetes and in Adults Aged 50 Years or Older: Two Randomized Controlled Trials. Journal of Medical Internet Research 21:e13363.

Poppe L, Van der Mispel C, De Bourdeaudhuij I, Verloigne M, Shadid S, and Crombez G. 2017. Users' thoughts and opinions about a self-regulation-based eHealth intervention targeting physical activity and the intake of fruit and vegetables: a qualitative study. Plos One 12:e0190020.

Qiu S, Sun H, Liu Y, Kanu JS, Li R, Yu Y, Huang X, Li B, and Zhang X. 2017. Prevalence and correlates of psychological distress among diabetes mellitus adults in the Jilin province in China: a cross-sectional study. PeerJ 5:e2869.

R Development Core Team. 2010. R: A language and environment for statistical computing. Vienna, Austria: R Foundation for Statistical Computing.

Peer) reviewing PDF | (2021:01:57126:1:0:CHECK 30 Apr 2021) 
520

521

522

523

524

525

526

527

528

529

530

531

532

533

534

535

536

537

538

539

540

541

542

543

544

545

546
Röcke C, Li S-C, and Smith J. 2009. Intraindividual variability in positive and negative affect over 45 days: Do older adults fluctuate less than young adults? Psychology and aging 24:863.

Rost S, Van Ryckeghem DM, Koval P, Sütterlin S, Vögele C, and Crombez G. 2016. Affective instability in patients with chronic pain: a diary approach. Pain 157:1783-1790.

Schwarzer R. 2008. Modeling health behavior change: How to predict and modify the adoption and maintenance of health behaviors. Applied Psychology 57:1-29.

Sigal RJ, Kenny GP, Wasserman DH, Castaneda-Sceppa C, and White RD. 2006. Physical activity/exercise and Type 2 diabetes A consensus statement from the American Diabetes Association. Diabetes Care 29:1433-1438.

Troiano RP, Berrigan D, Dodd KW, Mâsse LC, Tilert T, and McDowell M. 2008. Physical activity in the United States measured by accelerometer. Med sci sports Exerc 40. 10.1249/mss.0b013e31815a51b3

Van Dyck D, Herman K, Poppe L, Crombez G, De Bourdeaudhuij I, and Gheysen F. 2019. Results of MyPlan 2.0 on physical activity in older Belgian adults: randomized controlled trial. Journal of Medical Internet Research 21:e13219.

Van Ryckeghem DM, Crombez G, Goubert L, De Houwer J, Onraedt T, and Van Damme S. 2013. The predictive value of attentional bias towards pain-related information in chronic pain patients: a diary study. PAIN® 154:468-475.

Vetrovsky T, Omcirk D, Malecek J, Stastny P, Steffl M, and Tufano JJ. 2021. Morning fatigue and structured exercise interact to affect non-exercise physical activity of fit and healthy older adults. BMC geriatrics 21:1-10.

WHO. 2020. WHO guidelines on physical activity and sedentary behaviour. Geneva.

Zenk SN, Horoi I, Jones KK, Finnegan L, Corte C, Riley B, and Wilbur J. 2017. Environmental and personal correlates of physical activity and sedentary behavior in African American women: An ecological momentary assessment study. Women \& health 57:446-462. 
Table $\mathbf{1}$ (on next page)

Demographic characteristics of the participants 
1 Table 1

2 Demographic characteristics of the participants

\begin{tabular}{ll}
\hline Characteristics & Participants $(\mathrm{N}=38)$ \\
\hline Sex & $13(34.21)$ \\
$\quad$ N women (\%) & $25(65.79)$ \\
$\quad$ N men (\%) & $63.18(7.80) ; 50.00-81.00$ \\
Age in years, mean (SD); range & \\
Educational level & $20(52.63)$ \\
$\quad$ N high level of education (\%) & $18(47.37)$ \\
$\quad$ N low level of education (\%) & $19(50.00)$ \\
Retirement & $19(50.00)$ \\
$\quad$ N retired (\%) & $109.25(15.14) ; 75.55-155.75$ \\
$\quad$ N not retired (\%) & $30.82(6.00) ; 21.40-50.90$ \\
Waist circumference in cm, mean (SD); range & $129.40(83.31) ; 12.00-288.00$ \\
BMI (kg/m $\left.{ }^{2}\right)$ mean (SD); range & $10.18(4.01) ; 6.00-24.00$ \\
Time since diagnosis in months, mean (SD); range & $8.63(3.68) ; 6.00-23.00$ \\
Level of anxiety, mean (SD); range & \\
Level of depression, mean (SD); range & \\
\hline
\end{tabular}

3

4 


\section{Table 2 (on next page)}

Mean levels of sedentary behaviour and PA and mean scores on the mental and somatic stressors 
1 Table 2

2 Mean levels of sedentary behaviour and PA and mean scores on the mental and somatic

3 stressors

\begin{tabular}{|c|c|c|c|}
\hline Variable & mean (SD); range & $\begin{array}{l}\text { Between-subject } \\
\text { variance }\end{array}$ & $\begin{array}{l}\text { Within-subject } \\
\text { variance }\end{array}$ \\
\hline \multicolumn{4}{|l|}{ Sedentary behaviour and } \\
\hline \multicolumn{4}{|l|}{ PA ( $\%$ of wear time) } \\
\hline Sitting time & $\begin{array}{l}58.14(8.18) ; \\
40.84-75.23\end{array}$ & 57.39 & 64.75 \\
\hline LPA & $\begin{array}{l}35.54(7.18) \\
21.57-51.91\end{array}$ & 44.32 & 47.56 \\
\hline MVPA & $\begin{array}{l}6.31(2.82) \\
0.74-13.93\end{array}$ & 6.14 & 15.85 \\
\hline \multicolumn{4}{|l|}{ Stressors* } \\
\hline Fatigue & $\begin{array}{l}2.34(1.31) \\
1.00-6.50\end{array}$ & 1.54 & 1.63 \\
\hline Stress & $\begin{array}{l}1.81(1.12) \\
1.00-6.20\end{array}$ & 1.18 & 0.83 \\
\hline Pain & $\begin{array}{l}2.14(1.44) \\
1.00-6.80\end{array}$ & 2.01 & 0.67 \\
\hline Nausea/dizziness & $\begin{array}{l}1.22(0.57) \\
1.00-3.90\end{array}$ & 0.29 & 0.30 \\
\hline Numbness/tingling & $\begin{array}{l}1.42(0.85) \\
1.00-5.33\end{array}$ & 0.68 & 0.45 \\
\hline Sadness & $\begin{array}{l}1.37(0.70) \\
1.00-3.75\end{array}$ & 0.42 & 0.57 \\
\hline
\end{tabular}

4 Note. *Range of the items: 1 (absolutely not) to 10 (very much) 


\section{Table 3 (on next page)}

Results of the analysis for sedentary behaviour, LPA and MVPA 
1 Table 3

2 Results of the analysis for sedentary behaviour, LPA and MVPA

\begin{tabular}{|c|c|c|c|c|c|c|}
\hline & \multicolumn{2}{|c|}{ Sedentary behaviour } & \multicolumn{2}{|r|}{ LPA } & \multicolumn{2}{|c|}{ MVPA } \\
\hline & Beta & CI & Beta & CI & Beta & CI \\
\hline \multicolumn{7}{|l|}{ Within-subject } \\
\hline \multicolumn{7}{|l|}{ stressors } \\
\hline Fatigue & 0.38 & $-0.41 ; 1.26$ & -0.38 & $-1.03 ; 0.27$ & -0.005 & $-0.06 ; 0.05$ \\
\hline Stress & -0.07 & $-1.26 ; 1.10$ & 0.16 & $-0.77 ; 1.10$ & -0.04 & $-0.11 ; 0.04$ \\
\hline Pain & -0.43 & $-1.72 ; 0.85$ & 0.55 & $-0.44 ; 1.55$ & 0.02 & $-0.06 ; 0.11$ \\
\hline Nausea/dizziness & -1.07 & $-2.96 ; 0.94$ & 0.89 & $-0.82 ; 2.61$ & 0.02 & $-0.12 ; 0.15$ \\
\hline Numbness/tingling & 0.84 & $-0.72 ; 2.33$ & -0.72 & $-1.80 ; 0.36$ & -0.04 & $-0.15 ; 0.07$ \\
\hline Sadness & -0.26 & $-1.55 ; 1.00$ & 0.13 & $-0.99 ; 1.26$ & -0.01 & $-0.09 ; 0.07$ \\
\hline \multicolumn{7}{|l|}{ Between-subject } \\
\hline \multicolumn{7}{|l|}{ stressors } \\
\hline Fatigue & 1.54 & $-0.72 ; 3.81$ & -1.02 & $-3.80 ; 1.75$ & -0.03 & $-0.23 ; 0.16$ \\
\hline Stress & 1.24 & $-1.62 ; 4.14$ & -1.34 & $-5.04 ; 2.35$ & -0.09 & $-0.35 ; 0.17$ \\
\hline Pain & -0.40 & $-2.35 ; 1.61$ & 0.63 & $-1.78 ; 3.03$ & -0.02 & $-0.20 ; 0.15$ \\
\hline Nausea/dizziness & -6.00 & $-11.25 ;-0.87$ & 5.57 & $-0.96 ; 12.10$ & 0.17 & $-0.29 ; 0.63$ \\
\hline Numbness/tingling & -0.08 & $-3.24 ; 3.37$ & -0.49 & $-4.62 ; 3.64$ & 0.17 & $-0.12 ; 0.46$ \\
\hline Sadness & 1.17 & $-2.44 ; 4.73$ & -0.19 & $-4.63 ; 4.25$ & -0.15 & $-0.46 ; 0.17$ \\
\hline
\end{tabular}

3 Note. Results controlled for age, sex, waist circumference, level of education, retirement, level of anxiety 4 (PROMIS), and level of depression (PROMIS). 